\title{
Empleo del modelo animal en la formación en endoscopia diagnóstica y terapéutica de la vía biliar
}

\author{
J. Usón-Gargalloª, F.M. Sánchez-Margallo a , F. Soria-Gálvezª , J. Boix-Valverde b, \\ M. Fernández-Bermejo c', R. Latorre-Reviriego ${ }^{d}$, S. Climent-Peris e, V. Tejedo-Grafía ${ }^{a}$
}

\begin{abstract}
Objetivo. Mostrar nuestra experiencia en la formación en endoscopia terapéutica de la vía biliar en modelo animal. Materiales y métodos. Esta actividad formativa tiene una duración de 13 horas y comienza iniciando al alumno en las diferencias anatómicas de las especies que se emplean, la porcina y la canina, con respecto a la anatomía humana. Las prácticas consisten en habituar al endoscopista a la visión lateral del duodenoscopio; se realizan en un modelo porcino debido a que es más sencillo atravesar el esfínter pilórico, aunque no suele ser muy adecuado para la canulación de la papila duodenal. Tras superar la primera fase se utiliza el modelo canino, que permite adquirir las habilidades y destrezas propias de esta técnica. La evaluación de los resultados se realiza mediante una encuesta anónima. Resultados. El 76\% de los alumnos confirma que ha avanzado mucho con este entrenamiento, un $18 \%$ regular y un $6 \%$ poco. El $75 \%$ considera que, tras realizar esta actividad de formación, se encontraría capacitado para llevar a cabo la técnica en su hospital. El 94\% de los alumnos recomendaría esta actividad a sus compañeros. Conclusiones. El empleo del modelo animal en la formación en colangiopancreatografía retrógada endoscópica (CPRE)

initiating the trainee in anatomical differences with regard to human anatomy that occur between species that are used, the swine and canine. The practices consist of endoscopist to habituate the lateral view of duodenoscope, performing on pig model because it is easier to pass through the pyloric sphincter, although this is not very suitable for cannulation of the duodenal papilla. After passing the first stage, we used the canine model to acquire the skills and abilities specific to this technique. The performance evaluation is done through an anonymous survey. Results. $76 \%$ of students confirmed that they have made good progress with this training, $18 \%$ regular and $6 \%$ that shortly. $75 \%$ believed that after making this training would be found competent to carry out the technique in their hospitals. About $94 \%$ of students would recommend this activity. Conclusions. The use of animal model in training in endoscopic retrograde cholangiopancreatography (ERCP) permits the acquisition of basic skills needed for practice, as it enables the repetition of the maneuvers and continuing care. We believe that this mixed methodology could shorten the learning curve for ERCP and reduce iatrogenic in the early stages.
\end{abstract} permite la adquisición de habilidades básicas necesarias para la práctica, ya que posibilita la repetición de las maniobras y la tutela continuada. Consideramos que con esta metodología mixta se podría acortar la curva de aprendizaje de la CPRE y disminuiría la iatrogenia en las primeras fases.

Palabras clave. CPRE. Formación. Modelo animal.

\section{Animal model training in diagnostic and therapeutic biliary tract endoscopy teaching}

Aim. To show our experience in training in therapeutic endoscopy of the biliary tract in animal model. Materials and methods. This training activity has duration of 13 hours and starts
Key words. Animal model. ERCP. Teaching.

\section{Introducción}

Como es bien conocido, la formación de especialistas en endoscopia digestiva es un camino largo que necesita un programa bien estructurado (consulta de bibliografía, asistencia a cursos específicos, demostraciones en vivo, estudio de material didáctico en vídeo, colaboración en exploraciones, etc.) y donde la curva de aprendizaje en técnicas avanzadas no debería ser soportada por los pa-

\footnotetext{
Centro de Cirugía de Mínima Invasión Jesús Usón. Cáceres.

b Hospital Universitari Trias i Pujol. Badalona, Barcelona.

c Hospital San Pedro de Alcántara. Cáceres.

d Universidad de Murcia. Murcia.

e Universidad de Zaragoza. Zaragoza, España.
}

Correspondencia Dr. Federico Soria Gálvez. Centro de Cirugía de Mínima Invasión Jesús Usón. Ctra. N-521, km 41,8. E-10071 Cáceres.

\section{Fax \\ +34927181033. \\ E-mail \\ fsoria@ccmijesususon.com \\ Agradecimientos A M.I. Delgado Márquez $y$ al personal de anestesiología y quirófano del CCMIJU, por su inestimable ayuda en esta actividad de formación.}


cientes [1-3]. Por este motivo existen multitud de simuladores (maniquíes de plástico, vísceras de animales, animales anestesiados y simuladores virtuales); todos estos sistemas de entrenamiento tienen en mayor o menor medida influencia en la disminución de la curva de aprendizaje, interviniendo en la reducción del tiempo de las exploraciones y iatrogenias e incrementando la tasa de éxito en la práctica de las técnicas endoscópicas [4].

En este trabajo evaluamos nuestro procedimiento de formación en endoscopia terapéutica de la vía biliar en modelo animal, que realizamos desde hace 10 años.

\section{Materiales y métodos}

Esta actividad formativa en modelo animal se divide en dos fases y tiene una duración de 13 horas. La primera fase comienza con el conocimiento del nuevo material, que es diferente al empleado habitualmente, tanto el duodenoscopio como la pincería específica para estas técnicas. Dentro de esta primera fase es necesario el conocimiento de la anatomía comparada de las especies a emplear, la porcina y la canina, frente a la humana (Tabla).

La segunda fase coincide con el inicio de la formación endoscópica y se emplean ejemplares de la especie porcina de $30-40 \mathrm{~kg}$. El cerdo presenta una peculiaridad en el esfínter pilórico, un engrosamiento muscular, adiposo, conjuntivo, que recibe el nombre de torus pilórico. Por otro lado, la papila duodenal mayor, que recibe exclusivamente el conducto colédoco, se localiza en los primeros centímetros del duodeno. Esta topografía de la papila, tan próxima al píloro, dificulta su canulación y favorece que, durante los movimientos de aproximación, el endoscopio regrese al estómago. El duodeno descendente, donde se localiza la papila duodenal mayor, no es retroperitoneal, como en la especie humana, y queda sujeto por un amplio mesoduodeno $(9-10 \mathrm{~cm})$ que le permite cierta movilidad. La papila duodenal menor se localiza caudalmente, a $15-18 \mathrm{~cm}$ del píloro, y recibe el conducto pancreático accesorio, único conducto pancreático en el cerdo. Esta papila es plana y de difícil identificación. Por estas circunstancias empleamos la especie porcina únicamente para el aprendizaje del manejo del duodenoscopio, ya que las estructuras son mayores y existe menor dificultad para el paso del píloro con respecto a la especie canina.

\section{Tabla. Esquema del modelo de formación.}

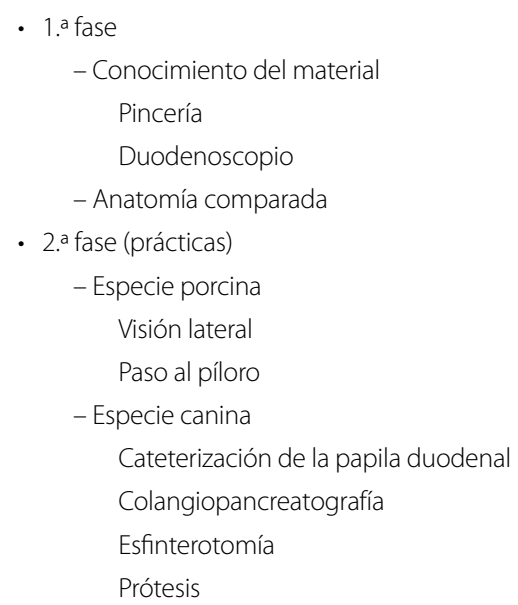

Tras la adquisición de las primeras destrezas endoscópicas (manejo del duodenoscopio y habituación a la visión lateral), el alumno comienza las prácticas con la especie canina. Los animales empleados son ejemplares de la raza Beagle de entre $15-20 \mathrm{~kg}$. En la especie canina, el conducto pancreático principal no es constante $y$, cuando se presenta, está poco desarrollado y drena, junto al colédoco, en la papila duodenal mayor. Dicha papila se localiza a mayor distancia del píloro que en el cerdo $(4-6 \mathrm{~cm})$, lo que favorece su canulación, a pesar de que el duodeno en esta especie es muy móvil al ser un órgano peritoneal y presentar un mesoduodeno amplio. El conducto pancreático accesorio es responsable del drenaje de la mayor parte de las secreciones pancreáticas y desemboca en la papila duodenal menor, localizada en el duodeno descendente, a 8-10 cm del píloro (Fig. 1).

La especie canina permite adquirir las habilidades y destrezas propias de esta técnica: canulación de la papila, colangiografía y control fluoroscópico, esfinterotomía, dilatación, empleo de cesta y sistemas de dilatación, colocación de prótesis, etc. (Fig. 2).

La evaluación de los resultados de esta actividad se realiza mediante una encuesta anónima donde se describen las destrezas adquiridas y la utilidad de esta actividad en el modelo animal.

Esta actividad ha sido aprobada por el comité de ética de experimentación animal y cumple la 


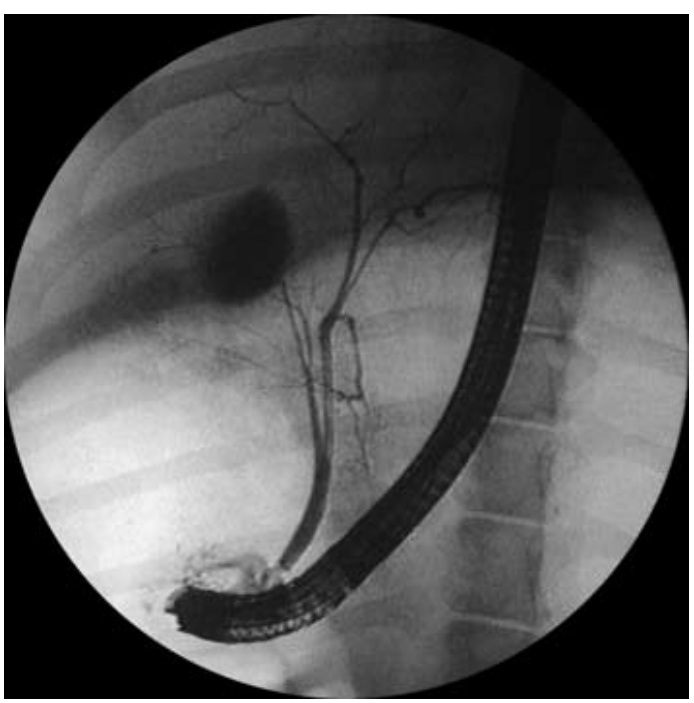

Figura 1. Colangiopancreatografía retrógrada endoscópica en un modelo animal.

Directiva de la UE 86/609/CEE, de 26 de noviembre de 1986, sobre protección de animales utilizados para experimentación y otros fines científicos y docentes, que se ha incorporado al ordenamiento jurídico español mediante el RD 223/1988, de $14 / 03$, por la que se establecen las normas de los establecimientos de cría, suministradores y usuarios de animales de experimentación de titularidad estatal, así como las de autorización para el empleo de animales en experimentos, y el RD 1201/2005, sobre protección de animales utilizados para experimentación y otros fines científicos.

\section{Resultados}

A esta actividad anual han asistido 37 alumnos, todos con un nivel de formación similar en endoscopia digestiva (65\% médicos internos residentes), por lo que a la hora de la evaluación del progreso no se encontraron diferencias. Tras la evaluación de las encuestas anónimas, el 76\% de los alumnos confirma que ha avanzado mucho con este entrenamiento, un $18 \%$ regular y un $6 \%$ poco. El $75 \%$ considera que, tras realizar esta actividad de formación, se encontraría capacitado para llevar a cabo la técnica en el ámbito de la clínica. El 94\% de los alumnos recomendaría esta

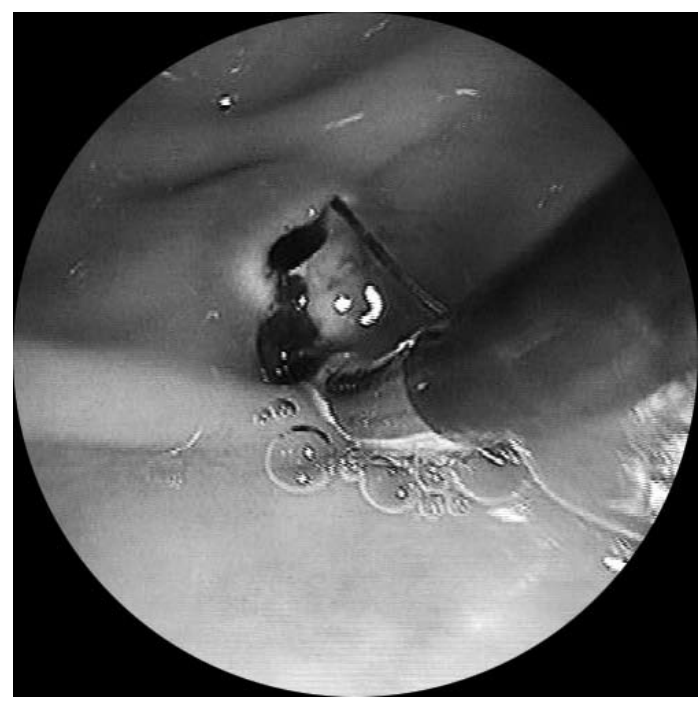

Figura 2. Esfinterotomía en un modelo animal.

actividad a sus compañeros, y el $6 \%$ restante no sabe o no contesta. Con respecto al tiempo necesario para superar el escollo que supone la visión lateral mostrada por el duodenoscopio y conseguir el paso a través del píloro, fue de media inferior a los 22 minutos. El tiempo necesario para conseguir la primera canulación fue inferior a 17 minutos. La colangiopancreatografía fue conseguida sólo por el $40,5 \%$ de los asistentes.

Ningún animal relacionado con esta actividad falleció a consecuencia de estas actividades de entrenamiento. Únicamente encontramos un caso en el que se produjo un desgarro del conducto colédoco, por la liberación de una prótesis biliar de tamaño inadecuado, que se resolvió con la intervención de los servicios veterinarios.

\section{Discusión}

Las necesidades de formación en endoscopia digestiva son claras, aunque actualmente no existe un criterio consensuado [3]. Con mayor razón son necesarias en técnicas como las que nos ocupan, donde es imprescindible una gran destreza y sólo existe un pequeño margen de error, ya que las complicaciones y las iatrogenias provocan graves consecuencias [5]. En concreto, la 
colangiopancreatografía retrógada endoscópica (CPRE) es un procedimiento endoscópico avanzado que requiere un considerable entrenamiento y experiencia para desarrollarla efectivamente y de modo seguro. Además, es el procedimiento rutinario más peligroso que realizan los gastroenterólogos, con una morbilidad del 5-10\% y una mortalidad del 0,1-1\% [6].

Con respecto a la formación de especialistas se hace hincapié, por parte de las diferentes sociedades gastroenterológicas, en la necesidad de una adecuada formación, que ha pasado desde un número pequeño de exploraciones en el pasado, 2550 CPRE en 1988 en Estados Unidos [7], a un número más adecuado en los últimos años, 180-200 CPRE en el mismo país. Estos cambios se deben a los trabajos de evaluación de la formación que se han ido realizando en las últimas décadas [8].

Sin embargo, las recomendaciones del número de procedimientos supervisados necesarios para alcanzar la suficiencia en estas técnicas constituyen un patrón demasiado heterogéneo, ya que varían dependiendo de la sociedad científica que los avale, del número de expertos adecuados por hospital -ya que las exploraciones deben tutorizarse siempre- y del volumen de pacientes sometidos a endoscopia de la vía biliar, circunstancia que puede retrasar la consecución de una curva de aprendizaje adecuada [9]. Por tanto, hay otras propuestas para evaluar el grado de destreza adquirido en CPRE para finalizar la formación, como son alcanzar un $80 \%$ de éxito en la canulación biliar [10]. Sobre este aspecto, un estudio evidenció que este porcentaje se conseguía tras realizar $350 \mathrm{ca}-$ teterizaciones, número bastante alejado de lo sugerido en anteriores estudios [11].

Recientemente, un trabajo que evalúa la visión de los médicos en formación en gastroenterología con respecto a la endoscopia concluye que la formación es variable en cantidad y calidad, que la supervisión es con frecuencia inadecuada o ausente, y que, por tanto, existen muchas oportunidades de mejora en este aspecto [12].

Durante los últimos 30 años se han desarrollado diferentes tipos de simuladores, incluyendo modelos animales, simuladores con piezas orgánicas (Erlanger) y simuladores virtuales. Los objetivos de los simuladores deben ser la aceleración de la curva de aprendizaje, la mejora de la formación en endoscopia para los principiantes, el mantenimiento de la competencia cuando los procedimientos endoscópicos no se realizan con regularidad, y el aprendizaje de nuevas técnicas intervencionistas [13].

Los distintos tipos de simuladores para aprendizaje endoscópico muestran diferencias en cuanto al realismo de las estructuras simuladas y a la utilidad de éstas para el aprendizaje de las técnicas. Los simuladores por ordenador son los que requieren una mayor inversión inicial, pero permiten la adquisición de destrezas básicas y avanzadas, evaluando de modo sistemático la curva de aprendizaje. El modelo animal es más realista a pesar de las diferencias anatómicas. Por último, los simuladores con piezas orgánicas en su interior son económicos, pero requieren mucha manipulación para la preparación del modelo [13]. En un estudio llevado a cabo por la American Society for Gastrointestinal Endoscopy, la simulación virtual es calificada como inferior frente al modelo animal in vivo y al simulador con piezas orgánicas, por falta de realismo visual, ausencia de sensación táctil y menor ergonomía endoscópica [14].

En lo concerniente al modelo animal, en aras de conseguir un modelo que permita el entrenamiento, se han empleado principalmente tres especies. En 1974, Falkenstein et al describieron el uso de la especie canina como modelo de entrenamiento, concluyendo que la cateterización de la papila duodenal simula la técnica y las maniobras desarrolladas para la CPRE en humanos, lo que permite la adquisición de destreza en el modelo de laboratorio [15]. El uso de primates también se ha evaluado; aunque existen evidentes diferencias entre el tamaño de un humano adulto y un babuino, la anatomía del tracto gastrointestinal y el sistema biliopancreático guardan gran semejanza y es posible realizar la CPRE [16]. El empleo del cerdo como modelo para la formación en CPRE ya fue descrito en 1995 por Noar [3]; aunque con diferencias anatómicas, esta especie ha demostrado su utilidad en la formación de técnicas diagnósticas y terapéuticas de la vía biliar, siendo posible la cateterización, esfinterotomía, disposición de stents, etc. [5]. Debido a las diferencias anatómicas en la encrucijada biliopancreática del modelo animal porcino y la especie humana, referenciadas en la bibliografía, en nuestro planteamiento de formación empleamos un modelo mixto de aprendizaje: en primer lugar, la especie porcina, que permite habituarse a la visión lateral característica del duodenoscopio y cuyo paso por el píloro es más sencillo que en la especie canina. Una vez adquiridas las destrezas básicas se puede 
acceder al modelo canino, con la posibilidad de canular la papila duodenal con mayor facilidad que en la especie porcina.

Las ventajas, a diferencia de los simuladores inanimados, que aporta la formación en modelo animal en endoscopia digestiva son claras: sensación de tejido natural, elasticidad, sangrado, permiten la repetición de maniobras hasta su correcta realización, y las estructuras orgánicas transmiten una sensación táctil igual a las que encontramos en la práctica clínica [17], aunque no hay que olvidar, por contra, los aspectos éticos, higiénicos y legales, así como el coste de personal y material que conllevan estas actividades. No se permite la simulación de lesiones, el equipamiento debe ser exclusivo para su uso en animales y el centro precisa la necesaria acreditación para la manipulación de animales de experimentación [14,17]. Otro aspecto a tener en cuenta es la selección de los tutores, que deben ser endoscopistas expertos no sólo en clínica, sino en modelo animal, para de este modo poder aportar a los asistentes su experiencia teórica y práctica en este modelo.

Los beneficios que proporciona el empleo del modelo animal mixto en la formación inicial en CPRE, como se demuestra en este trabajo, son claros porque permite la adquisición de habilidades básicas necesarias para la práctica en un entorno muy fiel, posibilitando la repetición de las maniobras y la tutela continuada. Consideramos que con esta metodología se acorta la curva de aprendizaje de la CPRE y disminuye la iatrogenia en las primeras fases de aprendizaje de esta técnica avanzada, permitiendo a los no iniciados en esta técnica una toma de contacto con el diferente instrumental y las primeras prácticas donde adquirir destreza.

Estamos de acuerdo con diferentes asociaciones médicas a la hora de valorar que los cursos prácticos de corta duración no constituyen una formación suficiente para acreditarse en estos procedimientos [18]. Sin embargo, estos cursos pueden proporcionar experiencia en diferentes técnicas diagnósticas y terapéuticas, y proporcionar al clínico la oportunidad de desarrollar habilidades específicas y familiarizarse con los equipos $\mathrm{y}$ accesorios necesarios.

En conclusión, el empleo del modelo animal en actividades de formación está justificado para lograr una menor iatrogenia y para disminuir la curva de aprendizaje. Es necesario un protocolo de formación reglado que optimice el tiempo dedicado al entrenamiento. Además, el aprendizaje de una técnica tan compleja y con tanta morbilidad como es la CPRE necesita la formación en un laboratorio experimental.

\section{Bibliografía}

1. Ladas SD, Malfertheiner P, Axon A. An introductory course for training in endoscopy. Dig Dis 2002; 20: 242-5.

2. Waye JD, Leicester RJ. Teaching endoscopy in the new millennium. Gastrointest Endosc 2001; 54: 671-3.

3. Noar MD. An established porcine model for animate training in diagnostic and therapeutic ERCP. Endoscopy 1995; 27: 77-80.

4. Rodríguez-García JI, Turienzo-Santos E, Vigal-Brey G, Brea-Pastor A. Formación quirúrgica con simuladores en centros de entrenamiento. Cir Esp 2006; 76: 342-8.

5. Gholson CF, Provenza MJ, Doyle JT, Bacon BR. Endosocpic retrograde sphincterotomy in swine. Dig Dis Sci 1991; 36: 1406-9.

6. Freeman ML, Nelson DB, Sherman S, Haber GB, Herman ME, Dorscher PJ, et al. Complications of endoscopic biliary sphincterotomy. N Engl J Med 1996; 335: 909-18.

7. Health and Public Policy Committee, American College of Physicians. Clinical competence in diagnostic endoscopic retrograde cholangiopancreatography. Ann Intern Med 1988; 108: 142-4.

8. Jowell PS, Baillie J, Branch MS, Affronti J, Browning CL, Philips B. Quantitative assessment of procedural competence. A prospective study of training in endoscopic retrograde cholangiopancreatography. Ann Intern Med 1996; 125: 983-9.

9. Chutkan RK, Ahmad AS, Cohen J, Cruz-Correa M, Desilets D, Dominitz JA, et al. ERCP core curriculum prepared by the ASGE Training Committee. Gastrointest Endosc 2006; 63: 361-76.

10. Wicks AC, Robertson GS, Veitch PS. Structured training and assessment in ERCP has become essential for the Calman era. Gut 1999; 45: 154-6.

11. Jowell PS, Baillie J, Branch MS, Affronti J, Browning CL, Bute BP. Quantitative assessment of procedural competence. A prospective study of training in endoscopic retrograde cholangiopancreatography. Ann Intern Med 1996; 125: 983-9.

12. Wells C, Inglis S, Barton R. Trainees in gastroenterol- 
ogy views on teaching in clinical gastroenterology and endoscopy. Med Teach 2009; 31: 138-44.

13. Ferlitsch A, Glauninger P, Gupper A, Schillinger M, Haefner M, Gangl A, et al. Evaluation of a virtual endoscopy simulator for training in gastrointestinal endoscopy. Endoscopy 2002; 34: 698-702.

14. Sedlack R, Petersen B, Binmoeller K, Kolars J. A direct comparison of ERCP teaching models. Gastrointest Endosc 2003; 57: 886-90.

15. Falkenstein DB, Abrams RM, Kessler RE, Jones B, Johnson G, Zimmon DS. Endoscopic retrograde cholangio- pancreatography in the dogs: a model for training and research. Gastrointest Endosc 1974; 21: 25-6.

16. Siegel JH, Korsten MA. ERCP in a nonhuman primate. Gastrointest Endosc 1989; 35: 557-9.

17. Ledro D. Simuladores de formación en la endoscopia digestiva. An Med Interna 2004; 21: 456-60.

18. Society of American Gastrointestinal and Endoscopic Surgeons (SAGES) Guidelines Committee. Guidelines for training in diagnostic and therapeutic endoscopic retrograde cholangiopancreatography (ERCP). Surg Endosc 2007; 21: 1010-1. 